\title{
More passive internal tibial rotation with posterior cruciate ligament retention than with excision in a medial pivot TKA implanted with unrestricted caliper verified kinematic alignment
}

\author{
Alexander J. Nedopil ${ }^{1}$ (D) Stephen M. Howell ${ }^{2} \cdot$ Maury L. Hull $^{2}$
}

Received: 4 July 2021 / Accepted: 3 December 2021 / Published online: 18 December 2021

(C) The Author(s) 2021

\begin{abstract}
Purpose Excision of the posterior cruciate ligament (PCL) is recommended when implanting a medial pivot (MP) total knee arthroplasty (TKA) to reduce the risk of limiting flexion by over-tensioning the flexion space. The present study determined whether PCL retention (1) limits internal tibial rotation and (2) causes anterior lift-off of the insert in $90^{\circ}$ flexion after implantation of an MP design with unrestricted caliper verified kinematic alignment (KA).

Methods Four surgeons implanted an MP TKA design with medial ball-in-socket and lateral flat tibial insert in ten freshfrozen cadaveric knees. Before and after PCL excision, trial inserts with medial goniometric markings measured the angular I-E tibial orientation relative to the trial femoral component's medial condyle in extension and at $90^{\circ}$ flexion, and the surgeon recorded the occurrence of anterior lift-off of the insert at $90^{\circ}$ flexion.

Results PCL retention resulted in greater internal tibial rotation than PCL excision, with mean values of $15^{\circ}$ vs $7^{\circ}$ degrees from maximum extension to $90^{\circ}$ flexion, respectively $(p<0.0007)$. At $90^{\circ}$ flexion, no TKAs with PCL retention and one TKA with PCL excision had anterior lift-off of the insert (N.S.).

Conclusions This preliminary study of ten cadaveric knees showed that PCL retention restored more passive internal tibial rotation than PCL excision with a negligible risk of anterior lift-off. However, in vivo analysis from multiple authors with a larger sample size is required to recommend PCL retention with an MP TKA design implanted with unrestricted caliper verified KA.
\end{abstract}

Keywords Total knee replacement · Total knee arthroplasty · Calipered · Posterior cruciate ligament · Tibial rotation · Insert thickness

Alexander J. Nedopil

nedopil@me.com

Stephen M. Howell

sebhowell@mac.com

Maury L. Hull

mlhull@ucdavis.edu

1 Department of Orthopaedic Surgery, König-Ludwig-Haus, University of Würzburg, Würzburg, Germany

2 Department of Biomedical Engineering, University of California at Davis, 451 E. Health Sciences Drive, Room 2303, Davis, CA 95616, USA

\section{Introduction}

An ongoing debate in total knee arthroplasty (TKA) is whether to retain or excise the posterior cruciate ligament (PCL). Because PCL excision predictably increases laxity in the flexion space an unpredictable amount, two categories of implant design evolved to compensate for flexion instability: the medial pivot (MP) and the posterior stabilized (PS) cam-and-post mechanism $[8,11,16,36]$. In addition, PCL excision is often used with mechanically aligned (MA) TKA to treat an over-tensioned PCL and flexion space as most femoral and tibial components deviate from the patient's prearthritic joint lines which can cause anterior lift-off of the insert at $90^{\circ}$ flexion $[12,16,32,36]$.

Although the increase in flexion space laxity from PCL excision is well studied, the adverse effect on internal rotation of the tibia with flexion after TKA is less clear. In the 
native (i.e., healthy knee), the inherent PCL tension drives the tibia's internal rotation with knee flexion, which obligatorily decreases the Q-angle optimizing the retinacular ligaments' tension that guides patellofemoral tracking during knee flexion $[4,7,18]$. In TKA, restoring native internal rotation of the tibia could minimize the risks of patellar tilt, lateral displacement, and anterior knee pain, which the patient needs to experience high satisfaction $[3,19]$.

For the native knee, the reported mean value of internal tibial rotation from maximum extension to $90^{\circ}$ flexion is $18^{\circ}$ $[4,5,10]$. When performing TKA, this arc of tibial motion is desirable.

Two signs of an over-tight flexion space are loss of passive internal rotation of the tibia relative to the femur and anterior lift-off of the insert. A trial insert goniometer can determine the tibial orientation relative to a longitudinal line on the trial femoral component's medial condyle. The difference in orientation between flexion angles computes internal-external (I-E) rotation over a motion arc (Fig. 1) $[20,23]$. The occurrence of anterior lift-off or 'booking' of the trial insert (or baseplate) indicates an over-tight flexion space (Fig. 2) [32].

Accordingly, this study of ten cadaveric knees evaluated a MP TKA design with a medial ball-in-socket and flat lateral insert implanted with unrestricted caliper verified kinematic alignment (KA), which restores the patient's pre-arthritic joint lines and native knee posterior laxity and medial and lateral tibial compartment forces [28, 29, 33-35]. The first
Fig. 1 Schematics show a left trial insert goniometer with an anteromedial scale in $5^{\circ}$ intervals. The I-E rotation of the tibia relative to a longitudinal line on the medial condyle of the trial femoral component (circles) was $3^{\circ}$ external in extension and $12^{\circ}$ internal at $90^{\circ}$ of flexion resulting in $15^{\circ}$ of internal rotation like the native knee. The medial pivot insert is composed of a medial ball-insocket and a lateral flat articular surface
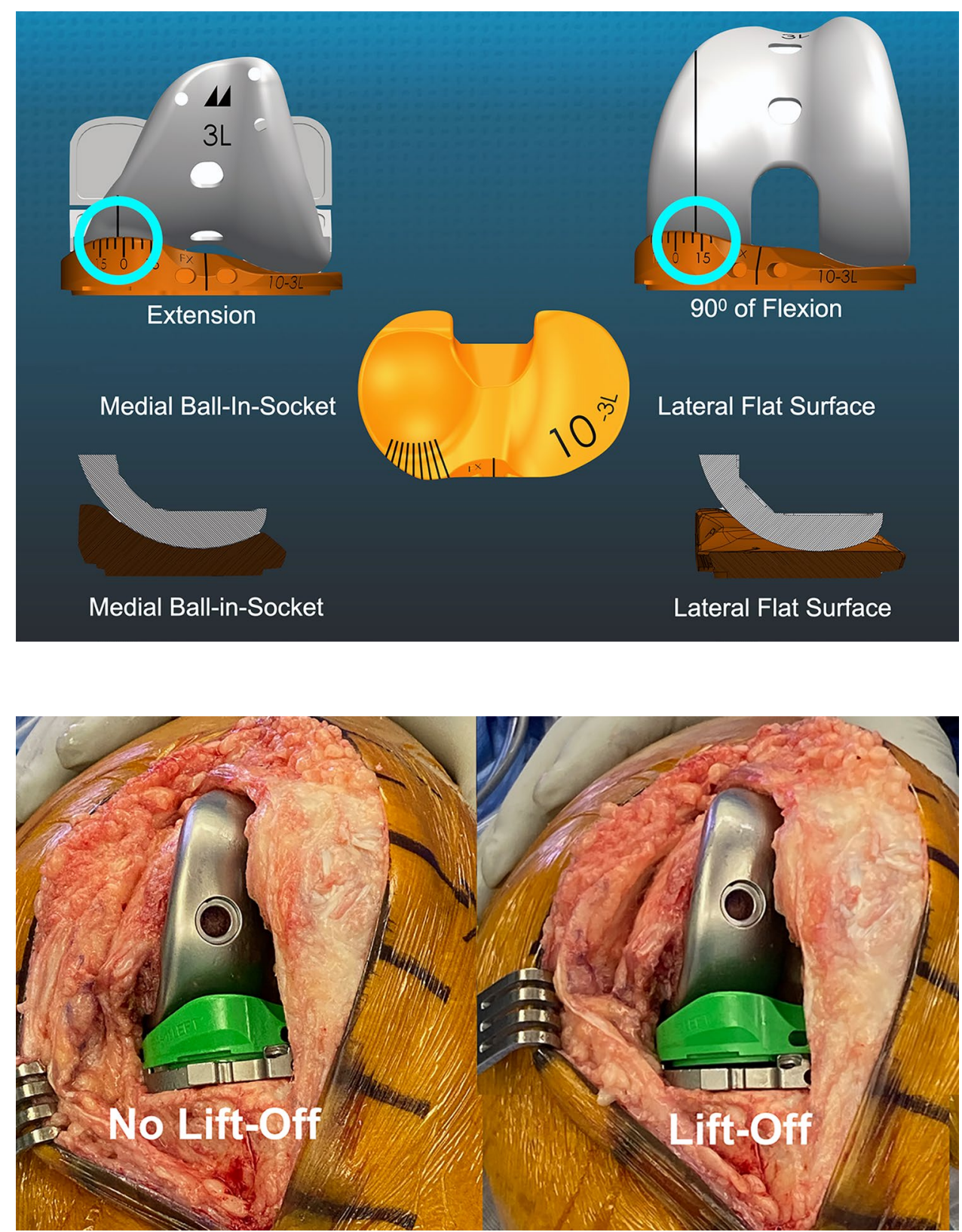

Fig. 2 Intraoperative photographs of a left TKA in $90^{\circ}$ flexion show examples of no anterior lift-off and lift-off of the trial insert (not goniometric) from the trial baseplate, which indicates an over-tensioned flexion space and PCL 
hypothesis was that PCL retention would restore internal tibial rotation between extension and $90^{\circ}$ flexion closer to reported mean values for the native knee than PCL excision. The second hypothesis was that PCL retention would not over-tension the TKA as indicated by a low occurrence of anterior lift-off of the insert at $90^{\circ}$ flexion. Proof of these hypotheses would support retaining the PCL when implanting the MP TKA design with unrestricted caliper verified KA.

\section{Methods and materials}

A registered tissue bank provided 12 fresh-frozen lower limb specimens from human donors for the study. An orthopedic surgeon screened anteroposterior and lateral radiographs of the knee and selected ten specimens that had no more than a Grade I or II Kellgren-Lawrence arthritis classification. One knee was studied in two female and eight male Caucasian donors with a mean age of $70 \pm 19$ years (29-88) and a mean BMI of $28 \pm 5 \mathrm{~kg} / \mathrm{m}^{2}$. Five knees were operated on by one orthopedic surgeon, two knees by another, and three knees by one of three surgeons. According to the University of California at Davis policies, this study did not require institutional review board (IRB) approval since the cadaveric specimens were de-identified. A power analysis determined that a matched pair analysis of nine specimens could detect a minimum difference of a $5^{\circ}$ change in internal rotation between TKA's with and without PCL retention assuming a Type I error (alpha) of 0.05 , a standard deviation of $5^{\circ}$ (i.e., effect size $=1$ ), and a power (1-beta) of 0.85 .

The study evaluated a MP TKA designed by Freeman and Pinskerova with a medial ball-in-socket conformity and a flat lateral insert (Fig. 1) [4, 5, 26, 27]. The insert had a posterior cut-out for retention of the PCLwith surrounding bone island. The tibial baseplate had an anatomic footprint that, when best-fit to the tibial resection, set the anterior-posterior (A-P) orientation parallel to the flexion-extension (F-E) plane of the pre-arthritic knee (GMK Sphere, Medacta International, www.medacta.com) [25]. The patella was left intact.

\section{Surgical technique}

The following is an overview of the previously described unrestricted caliper verified KA technique performed through a mid-vastus approach using intraoperatively recorded verification checks and following a decision-tree (Figs. 3, 4) [14]. For the femoral component, the varus-valgus $(\mathrm{V}-\mathrm{V})$ and $\mathrm{I}-\mathrm{E}$ orientations and the $\mathrm{A}-\mathrm{P}$ and proximal-distal (P-D) positions were set coincident with the patient's pre-arthritic distal and posterior joint lines by adjusting the calipered thicknesses of the distal and posterior femoral resections to within $0 \pm 0.5 \mathrm{~mm}$ of those of the femoral component condyles after compensating for cartilage wear and the kerf of the saw blade. An accuracy analysis showed these steps restore the distal lateral femoral joint line of $97 \%$ of patients within the normal left to right symmetry and set the I-E orientation of the femoral component with a deviation of $0.3^{\circ}$ (external) $\pm 1.1^{\circ}$ from the KA target of the F-E plane of the patient's knee [13, 21, 22, 24].

The surgeon followed six options in a decision-tree to set the $\mathrm{V}-\mathrm{V}$ and posterior slope orientation and thickness of the tibial component to restore the patient's pre-arthritic tibial joint line and limb alignment and balance the knee by restoring the native tibial compartment forces (Fig. 4) [20, 29, 33, 35]. The varus-valgus orientation of the tibial resection was adjusted working in $1^{\circ}-2^{\circ}$ increments until there was negligible medial and lateral lift-off from the femoral component during a varus-valgus laxity assessment in extension with the spacer block and trial tibial insert. An accuracy analysis showed that these steps restore the proximal tibial joint line of $97 \%$ of patients within native left to right symmetry [13, $15,24]$. The posterior slope was adjusted by setting an angel wing inserted through the medial slot of the tibial guide parallel to the patient's pre-arthritic slope. An accuracy analysis showed a $0^{\circ}$ mean difference between the tibial component's posterior slope and the patient's pre-arthritic posterior slope $[15,20]$. A best-fit of the largest anatomically shaped trial tibial baseplate inside the cortical rim of the tibial resection set the I-E orientation and A-P and medial-lateral (M-L) positions. An accuracy analysis showed a mean $2^{\circ}$ (external) $\pm 5^{\circ}$ deviation of the I-E orientation of the tibial component from the KA target of the F-E plane of the patient's knee [21, 24, 25, 28, 33, 35].

The following steps determined the optimal insert thickness. The knee was placed in $90^{\circ}$ of flexion and the PCL was palpated to verify that it was intact. A goniometric tibial insert that matched the thickness of the spacer block was positioned. The knee was placed in maximum extension and the surgeon verified that it hyperextended like the prearthritic knee. When the knee had a flexion contracture, a $1 \mathrm{~mm}$ thinner insert was used. The surgeon checked the $\mathrm{V}-\mathrm{V}$ laxity and verified that it was negligible in full extension and had a 3-4 mm lateral gap and negligible medial gap with the knee in $15^{\circ}-30^{\circ}$ of flexion. The knee was placed in $90^{\circ}$ of flexion and the surgeon verified that the passive I-E rotation of the tibia approximated $\pm 15^{\circ}$ like the native knee [30].

\section{Method for measuring the orientation of the tibia relative to the femoral component and recording anterior lift-off of the insert}

After inserting trial components, the surgeon used the back of the wrist to lift the heel and passively extend the knee without applying an I-E moment to the tibia. The 


\section{RECORD OF VERIFICATION CHECKS FOR CALIPERED KINEMATICALLY ALIGNED MEDACTA GMK SPHERE TKA}

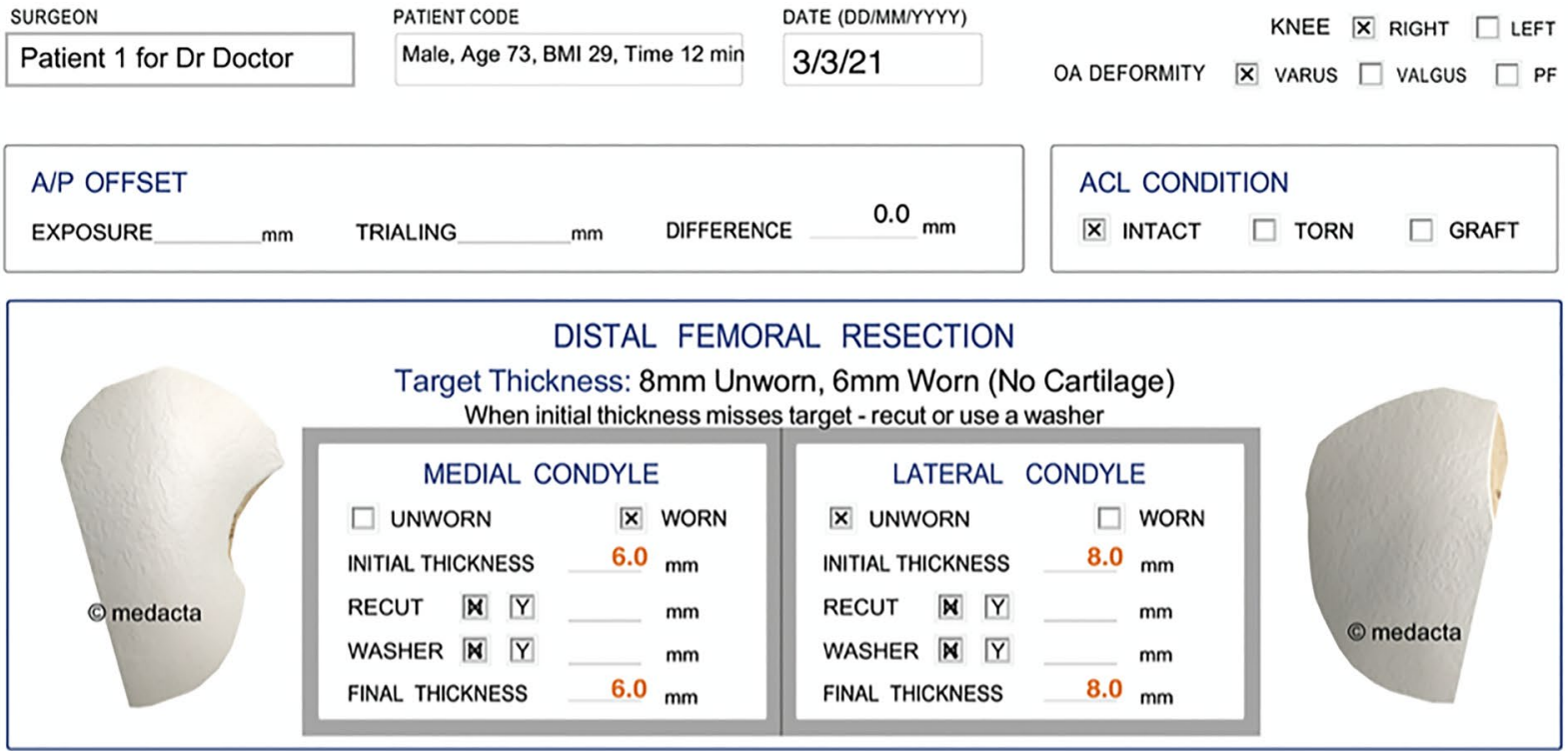
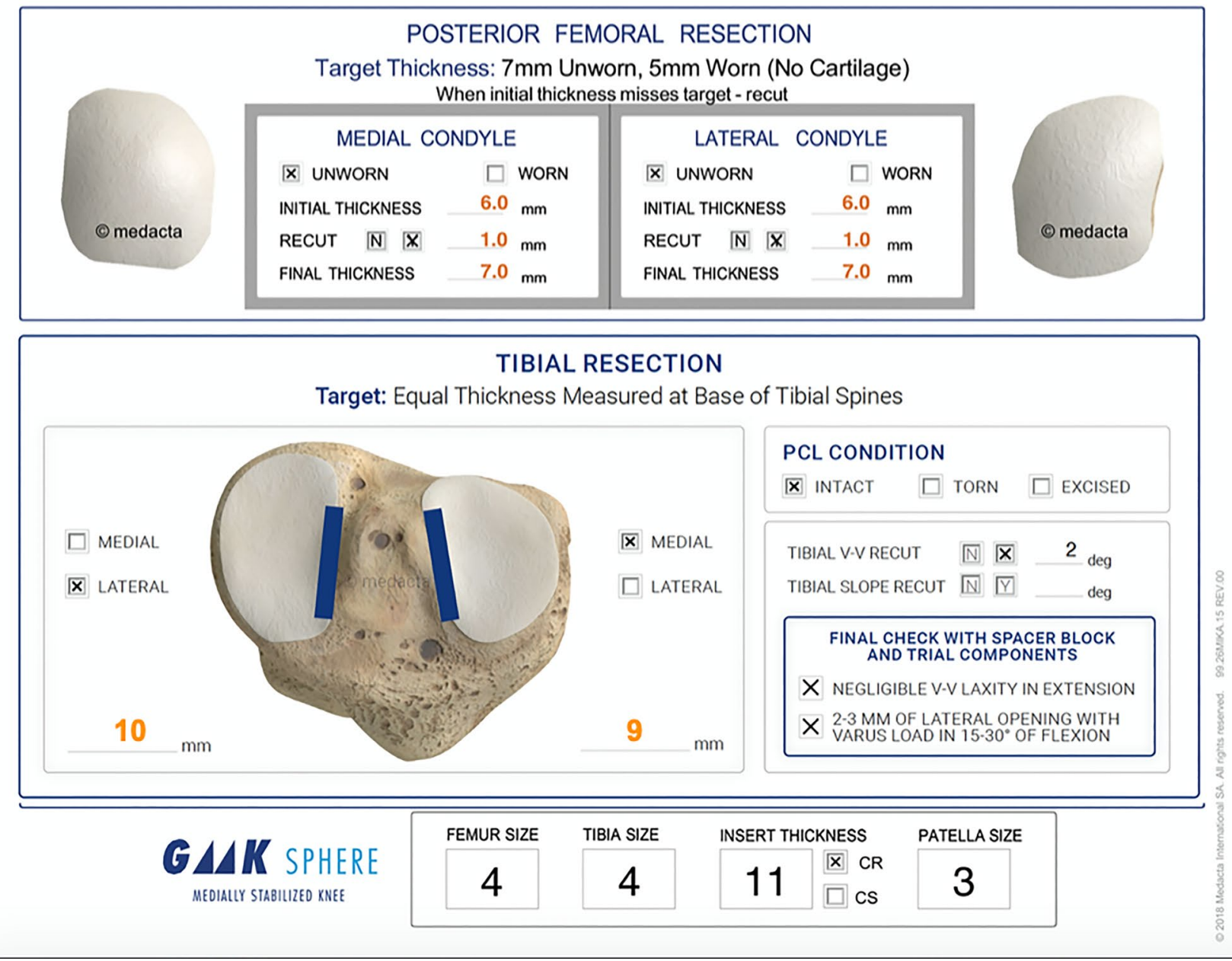
4Fig. 3 Worksheet for intraoperatively recording serial verification checks for a right knee with a varus deformity showing the caliper measurements of bone resections for a femoral component with a $9 \mathrm{~mm}$-thick distal femoral condyles and $8 \mathrm{~mm}$-thick posterior femoral condyles. The thicknesses of the distal and posterior femoral resections are adjusted so that they equal the thickness of the component within $0 \pm 0.5 \mathrm{~mm}$ after compensating for $2 \mathrm{~mm}$ of cartilage wear when present and $\mathrm{a} \sim 1 \mathrm{~mm}$ kerf from the saw cut

goniometric insert measured the angular tibial orientation relative to the line on the femoral component in degrees at extension (+ external/-internal). The surgeon reduced the patella, and the sub-vastus exposure stabilized the patella in the trochlear groove during flexion of the TKA to $90^{\circ}$. The heel was seated on a bump secured to the operating table, supporting the leg's weight. Without applying an I-E moment to the tibia, the surgeon recorded the tibial orientation of the insert goniometer relative to the femoral trial component and the occurrence of anterior lift-off as described by Scott [32]. When the insert trial lifted off the tibial baseplate anteriorly with knee flexion between $90^{\circ}$ to $100^{\circ}$, the test was positive, and the PCL and flexion space were too tight [32]. Lift-off was not measured in millimeters as no published reports describe this refinement of Scott's method [2].

The heel was seated on a bump secured to the operating table without applying an I-E moment to the tibia. With the foot resting on the operating table and supporting the leg's weight, the surgeon recorded the tibial orientation at $90^{\circ}$ flexion and the occurrence of anterior lift-off of the insert (Fig. 5). The surgeon recorded anterior lift-off as a dichotomous variable as either "absent" or "present".

\section{Statistical analysis}

Data were analyzed using statistical software (JMP ${ }^{\circledR}$ Pro 15.2.1, www.jmp.com, SAS, Cary, NC, USA). The mean and standard deviation described the continuous variables. A Student's paired $t$ test determined whether internal rotation of the TKA was different between PCL retention and excision. The Chi-square test determined whether the incidence of anterior lift-off of the insert was different between PCL retention and excision. Significance was $p<0.05$.

A senior academic bio-statistician recommended a repeatability and reproducibility analysis to characterize the measurement of tibial orientation relative to the femoral trial component with the insert goniometer. To characterize repeatability, a single surgeon measured the tibial orientation five times in four TKAs. The surgeon ranged the knee before measuring the tibial orientation in extension and $90^{\circ}$ flexion. Repeatability in extension and at $90^{\circ}$ flexion was computed as the pooled standard deviation and was $1^{\circ}$. To characterize reproducibility, two observers ( $\mathrm{SMH}$ and $\mathrm{AJN}$ ) measured the I-E orientation of the tibia at extension and $90^{\circ}$ flexion in seven knees. A two-way random-effects model analysis of variance (ANOVA) computed the intraclass correlation coefficient (ICC). An ICC value of $>0.9$ indicates excellent agreement, and 0.75-0.90 indicates good agreement. The ICC values of 0.89 for the measurement of tibial orientation at extension and 0.87 at $90^{\circ}$ of flexion indicated good reproducibility.

\section{Results}

Internal tibial rotation with PCL retention was greater than that with PCL excision. From extension to $90^{\circ}$ flexion, the mean internal tibial rotation was $15 \pm 5^{\circ}$ vs. $7 \pm 5^{\circ}$ for $\mathrm{PCL}$ retention vs PCL excision $(p<0.0007)$ (Fig. 6). At $90^{\circ}$ flexion, no TKA with PCL retention and one TKA with PCL excision had anterior lift-off of the insert (N.S.).

\section{Discussion}

The most important findings from the present study were that retaining the PCL with an MP TKA implanted with unrestricted caliper verified KA restored higher internal tibial rotation between extension and $90^{\circ}$ flexion and a negligible incidence of anterior lift-off of the insert when compared to PCL excision.

Although PCL retention is used sparingly with most MP designs because of concerns of limiting knee flexion, the present study showed that the PCL is needed to restore native internal tibial rotation and that its retention did not over-tension the flexion space. Similar to TKA, the function of the PCL in the native knee is crucial as cutting the PCL eliminates I-E tibial rotation [7]. Hence, promoting native knee internal tibial rotation also requires proper tension in the PCL. Fortunately, the restoration of native PCL tension is a direct consequence of unrestricted caliper verified KA TKA, as in vivo and in vitro studies reported restoration of native knee posterior laxity and medial and lateral tibial compartment forces [28, 29, 33-35].

Restoring native internal rotation about a medial pivot like the native knee requires the medial condyle of the femoral component to remain centered in the medial insert throughout the arc of motion, which may translate into favorable clinical results. A level 1 randomized-controlled trial reported that patients who underwent the medial constrained TKA scored significantly better on the Forgotten Joint Score and the quality-of-life subscale of the KOOS and KOOS-12 than those who underwent a CR-TKA [6]. These findings suggest that the medial constrained pivot knee is more likely to allow patients to "forget" the knee and restore their quality of life [6]. In vivo studies confirmed the medial pivot of the tibia during a deep knee bend and gait $[1,9,31]$. 
Fig. 4 Composite shows the decision-tree followed by the surgeons when they performed unrestricted caliper verified KA TKA. The technique sets the components to restore the pre-arthritic distal and posterior femoral and proximal tibial joint lines within $0 \pm 0.5 \mathrm{~mm}$, which restores native tibial compartment forces without the release of healthy ligaments, including the PCL [29, 33, 34]
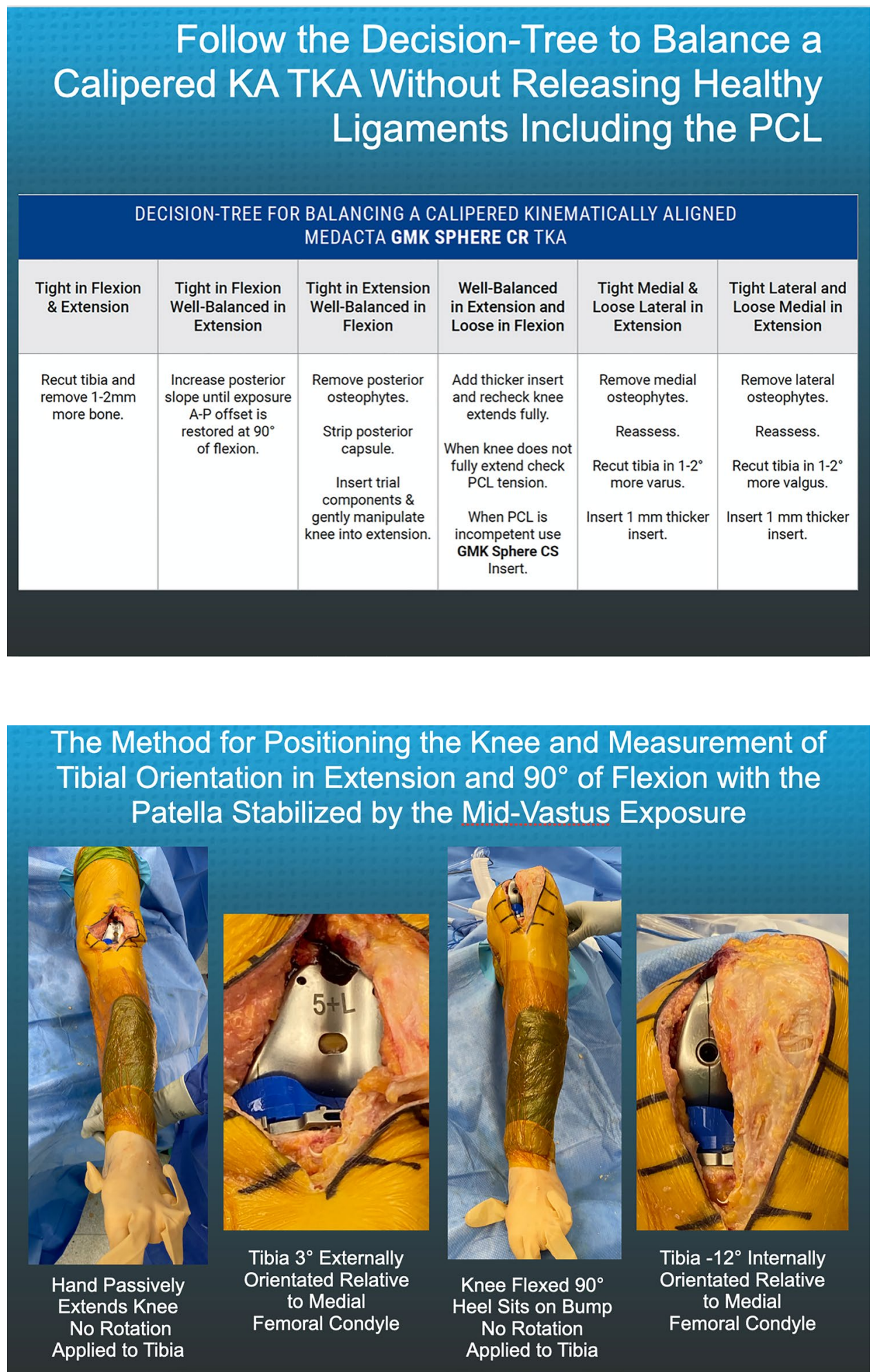

Fig. 5 Composite shows the method for positioning the TKA and measuring the angular tibial orientation of the goniometer insert relative to the femoral component with the knee passively extended and with the TKA in $90^{\circ}$ flexion with the heel resting on the bump supporting the leg without the surgeon applying rotation to the tibia
In addition, the lateral insert is flat to resemble the lack of lateral concavity in the native knee, which results in higher internal rotation than sagittal conforming lateral inserts with a posterior rim [1]. Thus, in the TKA, the posterior insert rim functions like a chock-block limiting internal tibial rotation [1]. In the native knee, the lateral meniscus does not limit internal tibial rotation since it falls off the posterior tibia in deep flexion [5, 17]. Hence, medial and lateral compartment articular geometry and PCL condition are critical factors governing tibial rotation relative to the femur [31]. 
Fig. 6 Box plots of ten cadaveric knees show the mean internal tibial rotation from maximum extension to $90^{\circ}$ of flexion (transverse line in the middle of the green diamond) of $15^{\circ}$ with PCL retention was significantly greater than the $7^{\circ}$ after PCL excision after implantation of a medial ball-in-socket and lateral flat tibial insert TKA with unrestricted calipered KA $(p<0.0007)$. The top and bottom edges of the green diamond indicate the $95 \%$ confidence interval limits

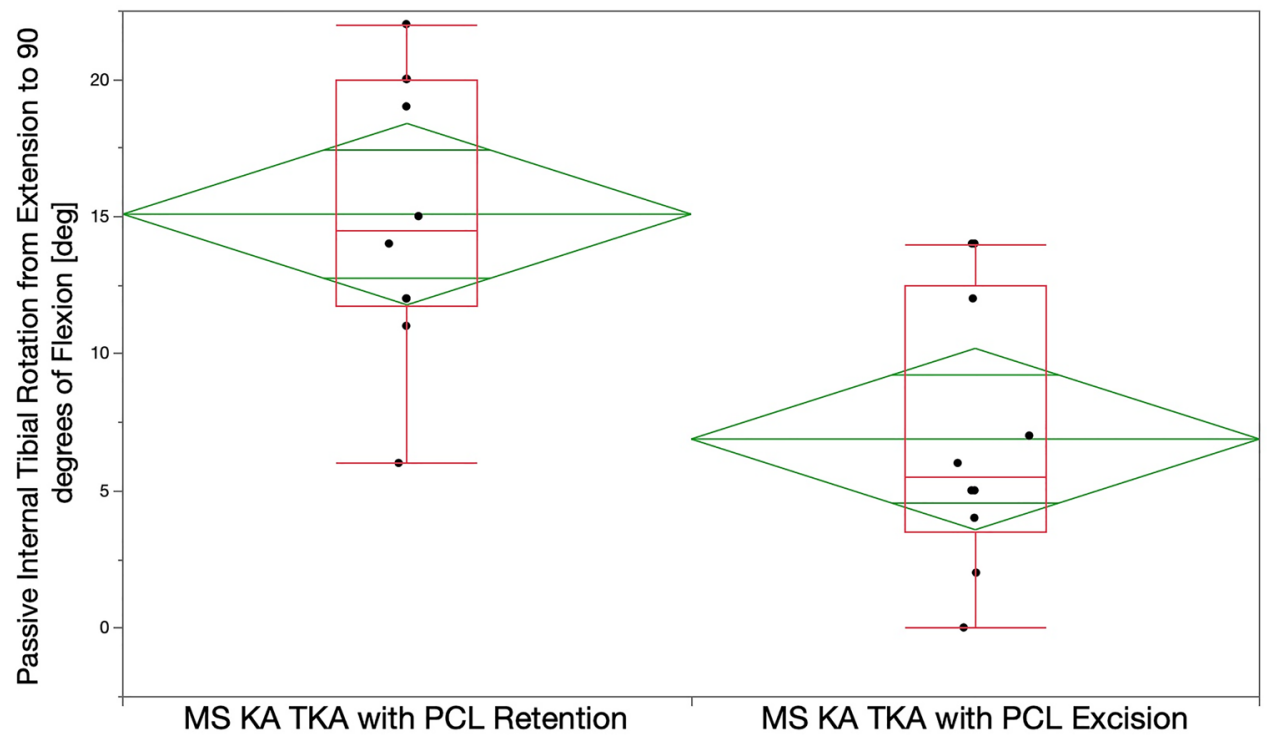

While PCL resection has the detrimental effect of predictably increasing laxity in the flexion space an unpredictable amount in TKA, the present study identified another undesirable kinematic consequence: the loss of half of the internal tibial rotation relative to the native knee at $90^{\circ}$ flexion. Like PCL resection in the native knee, no surgical strategy other than PCL reconstruction is likely to overcome the loss of internal tibial rotation from PCL resection in TKA and the concomitant risk of adverse clinical consequences such as patellar tilt, lateral displacement, and anterior knee pain [3, 7, 19].

The present cadaveric study has several limitations. The accuracy of the insert goniometer is specific for medial ball-in socket MP design and is not likely transferrable to a shallower and non-spherical MP design that allows medial A-P femorotibial displacement. The degree of internal tibial rotation is likely less for PS, PCL retaining, and ultra-congruent geometries, which have shallow medial and lateral insert concavities and a posterolateral rim that functions as a chock-block. The shallow medial concavity enables the femur to translate anteriorly and posteriorly, thereby lowering the PCL's tension necessary to drive internal tibial rotation. The degree of internal tibial rotation with PCL retention in a cadaveric knee in the present study could differ from in vivo values after unrestricted caliper verified KA TKA. Internal rotation values could change under different mechanisms of loading the TKA, such as weight-bearing activities.

Several factors could have affected the passive internal tibial rotation with PCL retention and excision. These factors included variance in the surgeon setting the slope of the tibial component which could tighten or loosen the flexion space and component size. However, because the study reported the difference in rotation within each knee specimen, these factors did not affect the primary conclusion that PCL retention restored more passive internal rotation than PCL excision.

\section{Conclusion}

This preliminary study of ten cadaveric knees showed that PCL retention restored more passive internal tibial rotation than PCL excision with a negligible risk of anterior liftoff. However, in vivo analysis from multiple authors with a larger sample size is required to recommend PCL retention with an MP TKA design implanted with unrestricted caliper verified KA.

Acknowledgements The authors would like to thank those who donated their remains to advance scientific study of TKA.

Author contributions All authors have made substantial contributions to all of the following: (1) the conception and design of the study, acquisition of data and analysis and interpretation of data, (2) drafting the article (3) final approval of the version submitted.

Funding Open Access funding enabled and organized by Projekt DEAL. Medacta International provided the cadaveric specimens, surgical instruments, and trial implants to perform the study.

\section{Declarations}

Conflict of interest A. J. Nedopil is a paid consultant for Medacta USA, Inc., S. M. Howell is a paid consultant for THINK Surgical and Medacta International., M. L. Hull receives research support from Medacta USA, Inc. 
Ethical approval This study did not require approval by the University of California Davis Institutional Review Board because de-identified specimens were used.

Open Access This article is licensed under a Creative Commons Attribution 4.0 International License, which permits use, sharing, adaptation, distribution and reproduction in any medium or format, as long as you give appropriate credit to the original author(s) and the source, provide a link to the Creative Commons licence, and indicate if changes were made. The images or other third party material in this article are included in the article's Creative Commons licence, unless indicated otherwise in a credit line to the material. If material is not included in the article's Creative Commons licence and your intended use is not permitted by statutory regulation or exceeds the permitted use, you will need to obtain permission directly from the copyright holder. To view a copy of this licence, visit http://creativecommons.org/licenses/by/4.0/.

\section{References}

1. Delman CM, Ridenour D, Howell SM, Hull ML (2021) The posterolateral upslope of a low-conforming insert blocks the medial pivot during a deep knee bend in TKA: a comparative analysis of two implants with different insert conformities. Knee Surg Sports Traumatol Arthrosc. https://doi.org/10.1007/ s00167-021-06668-8

2. Emerson RH Jr, Barrington JW, Olugbode SA, Alnachoukati OK (2016) A comparison of 2 tibial inserts of different constraint for cruciate-retaining primary total knee arthroplasty: an additional tool for balancing the posterior cruciate ligament. J Arthroplasty 31:425-428

3. Freedman BR, Brindle TJ, Sheehan FT (2014) Re-evaluating the functional implications of the Q-angle and its relationship to in-vivo patellofemoral kinematics. Clin Biomech (Bristol, Avon) 29:1139-1145

4. Freeman MA, Pinskerova V (2003) The movement of the knee studied by magnetic resonance imaging. Clin Orthop Relat Res. https://doi.org/10.1097/01.blo.0000063598.67412.0d35-43

5. Freeman MA, Pinskerova V (2005) The movement of the normal tibio-femoral joint. J Biomech 38:197-208

6. French SR, Munir S, Brighton R (2020) A single surgeon series comparing the outcomes of a cruciate retaining and medially stabilized total knee arthroplasty using kinematic alignment principles. J Arthroplasty 35:422-428

7. Fukubayashi T, Torzilli PA, Sherman MF, Warren RF (1982) An in vitro biomechanical evaluation of anterior-posterior motion of the knee. Tibial displacement, rotation, and torque. J Bone Jt Surg Am 64:258-264

8. Gaillard R, Lustig S, Peltier A, Villa V, Servien E, Neyret P (2016) Total knee implant posterior stabilised by a third condyle: design evolution and post-operative complications. Orthop Traumatol Surg Res 102:1061-1068

9. Gray HA, Guan S, Young TJ, Dowsey MM, Choong PF, Pandy MG (2020) Comparison of posterior-stabilized, cruciate-retaining, and medial-stabilized knee implant motion during gait. $\mathrm{J}$ Orthop Res 38:1753-1768

10. Hill PF, Vedi V, Williams A, Iwaki H, Pinskerova V, Freeman MA (2000) Tibiofemoral movement 2: the loaded and unloaded living knee studied by MRI. J Bone Jt Surg Br 82:1196-1198

11. Hino $\mathrm{K}$, Ishimaru M, Iseki $\mathrm{Y}$, Watanabe $\mathrm{S}$, Onishi $\mathrm{Y}$, Miura $\mathrm{H}$ (2013) Mid-flexion laxity is greater after posterior-stabilised total knee replacement than with cruciate-retaining procedures: a computer navigation study. Bone Joint J 95-B:493-497
12. Hirschmann MT, Hess S, Behrend H, Amsler F, Leclercq V, Moser LB (2019) Phenotyping of hip-knee-ankle angle in young non-osteoarthritic knees provides better understanding of native alignment variability. Knee Surg Sports Traumatol Arthrosc 27:1378-1384

13. Howell SM, Gill M, Shelton TJ, Nedopil AJ (2021) Reoperations are few and confined to the most valgus phenotypes 4 years after unrestricted calipered kinematically aligned TKA. Knee Surg Sports Traumatol Arthrosc. https://doi.org/10.1007/ s00167-021-06473-3

14. Howell SM, Shelton TJ, Gill M, Hull ML (2021) A cruciateretaining implant can treat both knees of most windswept deformities when performed with calipered kinematically aligned TKA. Knee Surg Sports Traumatol Arthrosc 29:437-445

15. Johnson JM, Mahfouz MR, Midillioglu MR, Nedopil AJ, Howell SM (2017) Three-dimensional analysis of the tibial resection plane relative to the arthritic tibial plateau in total knee arthroplasty. J Exp Orthop 4:27

16. Kayani B, Konan S, Horriat S, Ibrahim MS, Haddad FS (2019) Posterior cruciate ligament resection in total knee arthroplasty: the effect on flexion-extension gaps, mediolateral laxity, and fixed flexion deformity. Bone Jt J 101-B:1230-1237

17. Levy IM, Torzilli PA, Gould JD, Warren RF (1989) The effect of lateral meniscectomy on motion of the knee. J Bone Jt Surg Am 71:401-406

18. Li G, Gill TJ, DeFrate LE, Zayontz S, Glatt V, Zarins B (2002) Biomechanical consequences of PCL deficiency in the knee under simulated muscle loads - an in vitro experimental study. J Orthop Res 20:887-892

19. Mizuno Y, Kumagai M, Mattessich SM, Elias JJ, Ramrattan N, Cosgarea AJ et al (2001) Q-angle influences tibiofemoral and patellofemoral kinematics. J Orthop Res 19:834-840

20. Nedopil AJ, Delman C, Howell SM, Hull ML (2021) Restoring the patient's pre-arthritic posterior slope is the correct target for maximizing internal tibial rotation when implanting a PCL retaining TKA with calipered kinematic alignment. J Personal Med 11:516

21. Nedopil AJ, Howell SM, Hull ML (2020) Deviations in femoral joint lines using calipered kinematically aligned TKA from virtually planned joint lines are small and do not affect clinical outcomes. Knee Surg Sports Traumatol Arthrosc 28:3118-3127

22. Nedopil AJ, Howell SM, Hull ML (2016) Does malrotation of the tibial and femoral components compromise function in kinematically aligned total knee arthroplasty? Orthop Clin N Am 47:41-50

23. Nedopil AJ, Shekhar A, Howell SM, Hull ML (2021) An insert with less than spherical medial conformity causes a loss of passive internal rotation after calipered kinematically aligned TKA. Arch Orthop Trauma Surg 141:2287-2294

24. Nedopil AJ, Singh AK, Howell SM, Hull ML (2018) Does calipered kinematically aligned TKA restore native left to right symmetry of the lower limb and improve function? J Arthroplasty 33:398-406

25. Nedopil AJ, Zamora T, Shelton T, Howell SM, Hull M (2021) A best-fit of an anatomic tibial baseplate closely parallels the flexion-extension plane and covers a high percentage of the proximal tibia. J Knee Surg 34:1486-1494

26. Pinskerova V, Johal P, Nakagawa S, Sosna A, Williams A, Gedroyc W et al (2004) Does the femur roll-back with flexion? J Bone Jt Surg Br 86:925-931

27. Pinskerova V, Samuelson KM, Stammers J, Maruthainar K, Sosna A, Freeman MA (2009) The knee in full flexion: an anatomical study. J Bone Jt Surg Br 91:830-834

28. Roth JD, Howell SM, Hull ML (2019) Analysis of differences in laxities and neutral positions from native after kinematically aligned TKA using cruciate retaining implants. J Orthop Res 37:358-369 
29. Roth JD, Howell SM, Hull ML (2018) Kinematically aligned total knee arthroplasty limits high tibial forces, differences in tibial forces between compartments, and abnormal tibial contact kinematics during passive flexion. Knee Surg Sports Traumatol Arthrosc 26:1589-1601

30. Roth JD, Howell SM, Hull ML (2015) Native knee laxities at 0 degrees, 45 degrees, and 90 degrees of flexion and their relationship to the goal of the gap-balancing alignment method of total knee arthroplasty. J Bone Jt Surg Am 97:1678-1684

31. Schutz P, Taylor WR, Postolka B, Fucentese SF, Koch PP, Freeman MAR et al (2019) Kinematic evaluation of the gmk sphere implant during gait activities: a dynamic videofluoroscopy study. J Orthop Res 37:2337-2347

32. Scott RD, Chmell MJ (2008) Balancing the posterior cruciate ligament during cruciate-retaining fixed and mobile-bearing total knee arthroplasty: description of the pull-out lift-off and slideback tests. J Arthroplasty 23:605-608

33. Shelton TJ, Howell SM, Hull ML (2019) Is there a force target that predicts early patient-reported outcomes after kinematically aligned TKA? Clin Orthop Relat Res 477:1200-1207
34. Shelton TJ, Howell SM, Hull ML (2019) A total knee arthroplasty is stiffer when the intraoperative tibial force is greater than the native knee. J Knee Surg 32:1008-1014

35. Shelton TJ, Nedopil AJ, Howell SM, Hull ML (2017) Do varus or valgus outliers have higher forces in the medial or lateral compartments than those which are in-range after a kinematically aligned total knee arthroplasty? Limb and joint line alignment after kinematically aligned total knee arthroplasty. Bone Jt J 99-B:1319-1328

36. Warth LC, Deckard ER, Meneghini RM (2021) Posterior cruciate ligament resection does not consistently increase the flexion space in contemporary total knee arthroplasty. J Arthroplasty 36:963-969

Publisher's Note Springer Nature remains neutral with regard to jurisdictional claims in published maps and institutional affiliations. 